\title{
Multi-Stove Scheduling for Sustainable On-Demand Food Delivery
}

\author{
Tao Dai * and Xiangqi Fan * \\ Glorious Sun School of Management, Donghua University, Shanghai 200051, China \\ * Correspondence: t.dai@dhu.edu.cn (T.D.); 2201521@mail.dhu.edu.cn (X.F.)
}

Citation: Dai, T.; Fan, X. Multi-Stove Scheduling for Sustainable On-Demand Food Delivery. Sustainability 2021, 13, 13133. https:// doi.org/10.3390/su132313133

Academic Editors: Bin Shen, Tijun Fan and Haijun Wang

Received: 21 October 2021

Accepted: 23 November 2021

Published: 26 November 2021

Publisher's Note: MDPI stays neutral with regard to jurisdictional claims in published maps and institutional affiliations.

Copyright: (c) 2021 by the authors. Licensee MDPI, Basel, Switzerland. This article is an open access article distributed under the terms and conditions of the Creative Commons Attribution (CC BY) license (https:// creativecommons.org/licenses/by/ $4.0 /)$.

\begin{abstract}
Ordering food through mobile apps and crowdsourcing resources has become increasingly popular in the digital age. Restaurants can improve customer satisfaction to satisfy on-demand food orders by shortening waiting time and achieving sustainability through fuel reduction. In the present study, we construct a double-layer scheduling model, which is developed using the characteristics of on-demand food preparation, including the use of multiple stoves, a variety of dishes in one order, and the integration of the same dishes from different customers. The bottom layer is a multi-stove dish package scheduling model based on parallel machine scheduling. The upper layer is an order selection model based on the knapsack problem. To identify the optimal solution, four strategies for calculating the weight coefficient of the dish package are proposed to shorten the waiting time and realize sustainability. Numerical experiments are designed to analyze the differences of the final scheduling results under the four strategies. The bottom layer is extended to another model based on the vehicle routing optimization model, given the switch time between different dishes. The extension of the model is also compared in the numerical experiments. Our paper confirms the necessity of using a double-layer model for multi-strategy comparison in order to achieve sustainable on-demand scheduling.
\end{abstract}

Keywords: cooking scheduling; multiple stoves; mergeable products; sustainability

\section{Introduction}

The digital age has brought a qualitative breakthrough to the restaurant industry with the development of a catering takeaway platform. Takeaway ordering apps have been made available in different countries. For example, Deliveroo and JustEat are popular in European countries, Uber Eats and Grubhub are popular in North America, and Eleme and MeiTuan are popular in China. These online service apps facilitate the smooth transfer of information between demand from customers and supply from restaurants. Fast-developing platforms extend the service scope and add potential customers to the catering enterprises. These apps increase the business space of restaurants and, given the increasing number of competitors, elevate operation efficiency.

The SDG Report 2020 stated: "Sustainable development has been defined as development that meets the needs of the present without compromising the ability of future generations to meet their own needs. For sustainable development to be achieved, it is crucial to harmonize three core elements: environmental protection, economic growth and social inclusion." As an industry that supports people's livelihoods, the catering industry also pays special attention to sustainable development. First, improving the speed of meal scheduling while controlling the quality of food can reduce idle time in stove processing and the waiting time of distribution couriers. This action can save natural gas consumption and the power consumption of the courier. Second, restaurants face a typical fluctuating demand during peak time. Thus, an efficient production arrangement is key for catering enterprises to attract more potential customers, improve customer satisfaction, and realize revenue growth. Finally, part of the social responsibilities of catering enterprises are ensur- 
ing that orders are satisfied on time and avoiding prolonged working hours of employees in the high-temperature kitchen.

Figure 1 shows that online ordering is treated as a chain of processes, similar to a supply chain. Online ordering involves preparation and delivery. The process starts when an order arrives. The restaurant needs to receive the order in time and schedule the preparation of dishes. The courier will then pick up the prepared dishes and deliver them to corresponding customers. Existing practical and academic research pays particular attention to the delivery process. For instance, takeout route planning models and continuous optimization of algorithms are studied in order to improve the efficiency of delivery processes. To ease the tension of transportation capacity, part-time couriers convene through crowdsourcing and are added to the distribution system during peak hours. To ensure the speed of distribution and sufficient capacity, the takeout platforms are devoted to finding an appropriate reward system. These efforts contribute to the efficient capacity of the delivery process. However, compared with the delivery process, the food preparing process is harder to adjust during peak periods because of poor flexibility. The outbreak of COVID-19 left a huge impact on people's daily lives. The increasing online takeout need leads to the constant consideration of waiting time for consumers. A survey on the "external factors affecting the delivery time of takeout couriers" for couriers showed that "slow meal preparing speed of merchants" was on the top of the list, at $70.27 \%$; this result shows that this item is the most critical factor that affects order completion time [1]. Additionally, restaurants are facing double pressures from online orders and in-store costumers. If online orders cannot be completed on time, the restaurant will not only be pressured and face complaints from couriers and online customers, but they also run the risk of losing in-store customers with the dropping service expectations. Therefore, focusing on the food preparation process is critical to increasing the sustainability of the whole industry.

$\longrightarrow$ Information Flow $\longrightarrow$ Logistic Flow

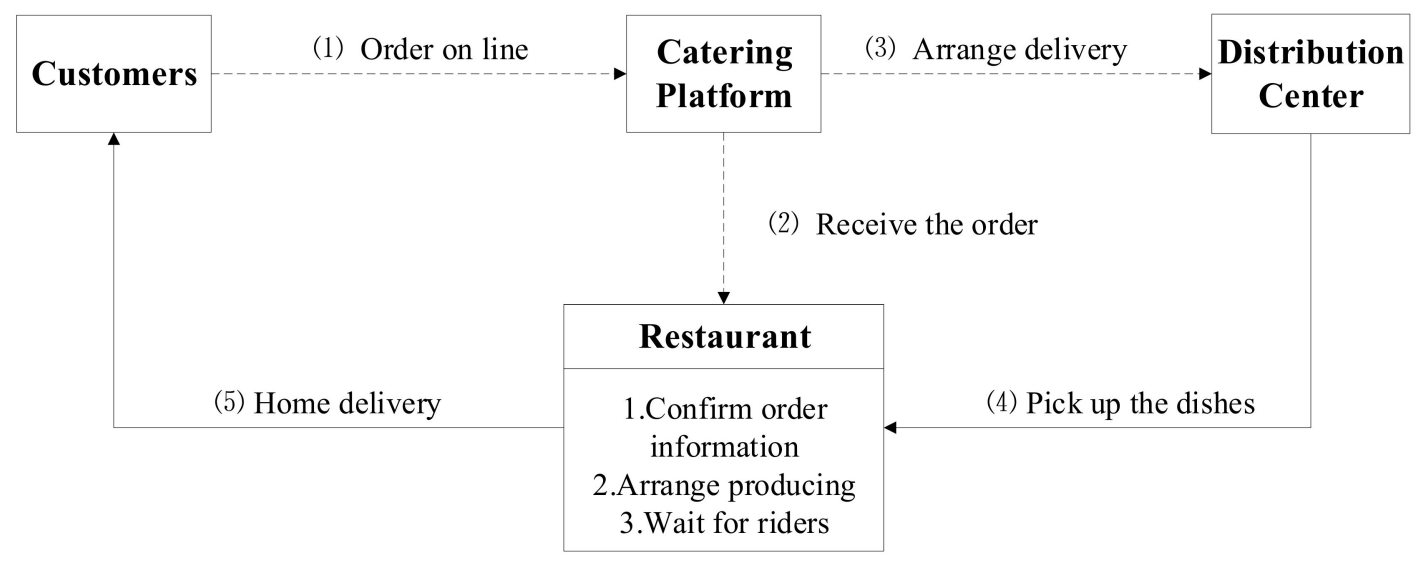

Figure 1. "Supply chain" network of catering enterprises.

The real meal scheduling problem is summarized as follows. Each order includes different dishes at varying quantities, each with an expected completion time according to the arrival of courier and the distance between the restaurant and customer. Restaurants use several identical stoves that can process a batch of the same dishes with corresponding size limitations. Different dishes may be prepared for a certain switch time. A multi-stove double-layer scheduling model with product merging is constructed according to order demand and production characteristics. In the model, the bottom layer implements the parallel machine scheduling of the dish-package, and the upper layer realizes the optimal selection of the customer order and the dish-package.

The novelty of the present paper is the attempt to convert the complex takeout problem into a two-level scheduling problem. To reduce deviation from the original problem, we 
introduce a weight coefficient to the dish-package and design four calculation strategies (scheduling strategy). We noted a similarity between the vehicle routing optimization (VRP) model and the dish-package scheduling model when switch time was considered. Therefore, VRP-based modeling is applied.

The remainder of this paper is organized as follows. Section 2 presents an exhaustive literature review. Section 3 defines the problem and proposes a double-layer scheduling model. Four scheduling strategies are compared through an example in Section 4. Section 5 proposes a new model that considers switch time. The four scheduling strategies are compared with the new scheduling scenarios. Finally, a conclusion that summarizes the main findings and future research directions are presented.

\section{Literature Review}

Existing research on the optimization of online takeout orders mainly focuses on distribution. Liu et al. [2] pointed out that online takeout ordering and delivery is an emerging service that can facilitate the delivery of take-out food by assigned staff after users order online. The study built a food delivery network to support distribution by leveraging spatial crowdsourcing. However, the system has room for improvement, particularly on the side of food preparation. Liu et al. [3] found that customers are sensitive to waiting, and the length of waiting time will directly affect customers' evaluations of the service.

Figure 2 gives an overview of present papers related to this paper.

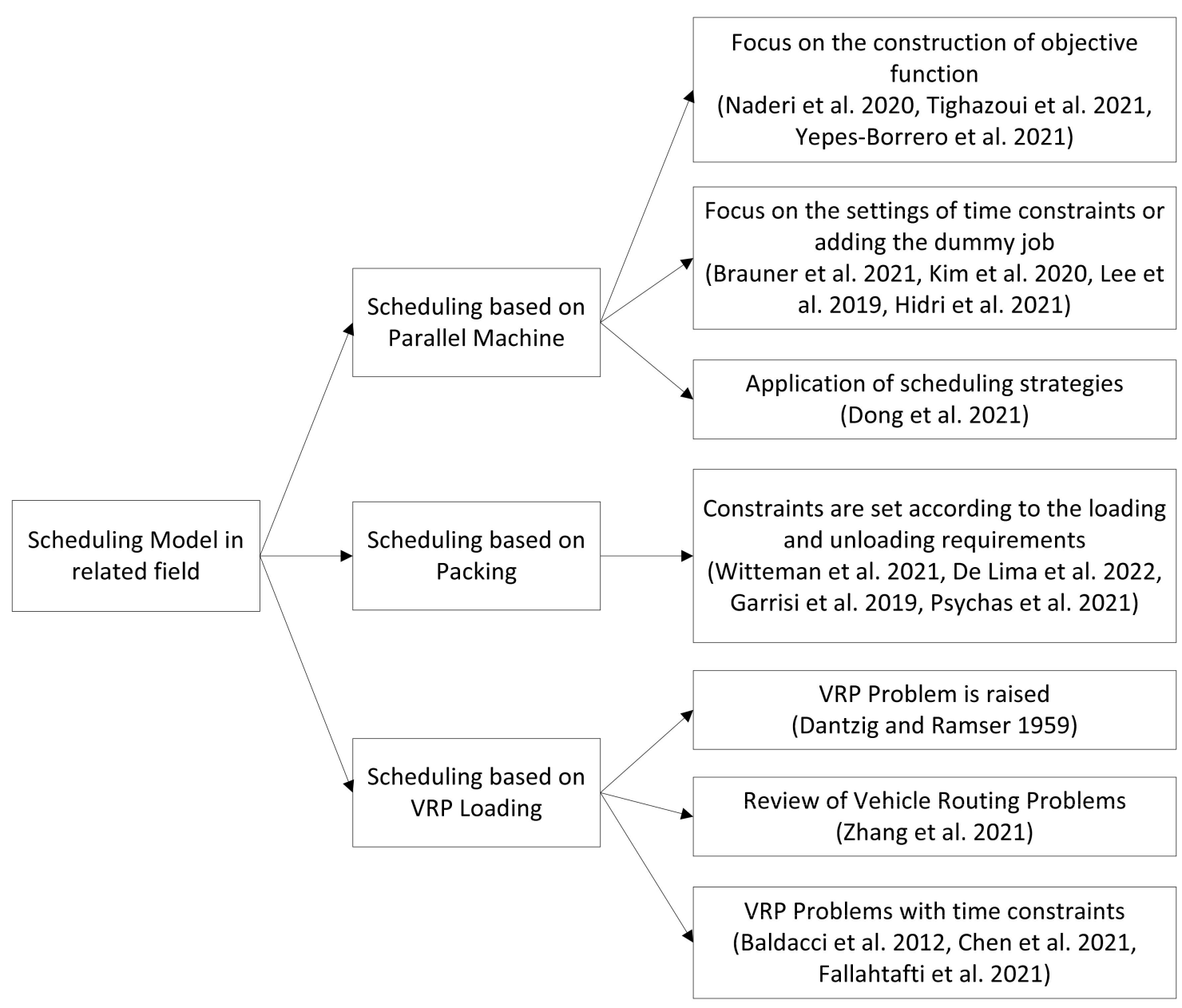

Figure 2. Literature review of scheduling model.

In the parallel machine scheduling papers, Naderi et al. [4] showed that order acceptance and the identical parallel machine scheduling problem are NP-hard; researches 
in this direction should focus on the description of the model. For example, Brauner et al. [5] used a small square to represent the processing of one job in the corresponding unit-time interval. Expressing all time interval with this small square solves the schedule problem with the release dates and deadlines. Tighazoui et al. [6] proposed the idea of measuring efficiency by the total weighted waiting times and stability on a single machine rescheduling problem. The authors extend decision-making from the makespan to the weighted sum of waiting time. According to the production characters and different concerns, Kim et al. [7] examined a 3D printer-based parallel machine scheduling problem with multiple processing alternatives and sequence-dependent setup times. Yepes-Borrero et al. [8] studied a bi-objective parallel machine scheduling problem with minimization of the makespan and the number of additional resources. Lee et al. [9] examined a uniform parallel machine scheduling problem and pointed to the need for a constraint in the first jobs on these machines because setup times for those jobs are ignored. Hidri et al. [10] solved this question by proposing that the first processed job in each machine has as immediate predecessor, namely, the dummy job. In addition, Dong et al. [11] reviewed several scheduling strategies, such as the first-in-first-out strategy, fair strategy, capacity strategy, and flex strategy.

Stove scheduling also involves the allocation of dishes. This problem is similar to the knapsack problem (packing problem), which has been examined in many studies. Witteman et al. [12] solved the aircraft maintenance task allocation problem by dividing the planning horizon into variable size bins, which also involves adding deadlines, intervals, and arrivals. De Lima et al. [13] reviewed the foundations and applications of network flow formulations. The constraints in different studies of the packing problem are set according to the loading and unloading requirements. Garrisi et al. [14] studied a train scheduling problem, which was provided as a mixed integer linear program; they also set variables to avoid collisions between two trains passing through the same section. Psychas et al. [15] studied the high-throughput bin packing problem with scheduling jobs that have random resource demands by initially characterizing a fundamental limit on the maximum throughput in such a setting.

The scheduling problem bears similarity with VRP. This classic problem was raised by Dantzig and Ramser [16] in 1959, with abundant research results. The existing related modeling and algorithm can be used for reference. Zhang et al. [17] reviewed the research on the VRP and pointed out that the VRP model can be used to optimize the economy or a service under certain constraints while serving all demand points. In a study with delivery time as the constraint, Baldacci et al. [18] examined a distribution service with a hard time window, which means that the arrival time must be within a limited time range. A fleet of $\mathrm{m}$ identical vehicles of capacity $\mathrm{Q}$ is stationed at the depot and these could fulfill customer demands. Chen et al. [19] also defined the different types of time when studying the robot workshop handling problem with the time window. Fallahtafti et al. [20] dealt with the selective pickup and delivery problems of a multi-stage network supply chain. The authors appropriately modelled time slot allocation to that of trucks by considering the type of trucks, arrival time to the plant warehouse, number of time slots required for unloading, and availability of warehouse time slots.

In summary, the takeaway food delivery chain can be divided into two processes, and a huge gap exists between the cooking and delivery scheduling process, which is the main purpose of the present paper. Our paper is built on existing research in the fields of parallel machine scheduling, packing scheduling, and VRP. A double-layer scheduling model that can be directly applied is constructed. Four tendentious scheduling strategies for calculating the weight coefficient of the dish-package are designed. The catering enterprises can use the decision-making model and method to deal with the production problem in peak periods and improve meal cooking efficiency. 


\section{Double-Layer Scheduling Model}

\subsection{Problem Description}

The problem can be formally stated as follows. Order $w$ with $p$ kinds of choices and corresponding quantities exist. The demand for the orders is grouped into batches called the dish-package. Each package is scheduled on $\mathrm{m}$ identical batch processing stoves. To shorten the final completion time of the customer order, the restaurant exerts effort to ensure that all dishes are ready before the courier arrives at the store. Therefore, the estimated arrival time of the courier is set as the expected cooking completion time of the order.

The types and quantities of all dishes are counted when a restaurant receives a batch of orders. The dishes are then combined into a dish-package. The limitation of mergeable dishes is different. If the total number exceeds the limitation, a new group of packages will be created. Only one dish-package can be processed for each stove at a time. Two decisions are implicitly needed for the problem:

1. Arrangement of dish-packages on the stoves.

2. Allocation of dish-packages to needed orders. The two-phase scheduling aims to ensure that the completion time of the order is earlier than the expected time.

\subsection{Logic of the Double-Layer Model}

We construct a double-layer model according to the two-phase decision. The input of the bottom layer (foundation layer) model is the number of dish-packages with their weight coefficient. The output is the stove scheduling and completion time of each dish-package. The completion time of the dish-package is the input of the upper layer, whereas the output is the completion time of the whole order, which is determined by the latest finished dish.

Given the structure of the double-layer, the completion time of the final order is completely influenced by the first-stage stove scheduling. Therefore, we designed four scheduling strategies for the first-stage decision. The strategies are carried out by the weight coefficients added on each initial dish-package. The four scheduling strategies are shown in Table 1.

Table 1. Weight coefficient calculation strategy.

\begin{tabular}{ccc}
\hline Strategy & Objectives of the Strategy & Value-Setting \\
\hline No. 1 & Workload balance between stoves & Set every dish-package as the same weight \\
No. 2 & The dish with the shortest cooking is taken first & Take the reciprocal of the cooking time as the weight \\
No. 3 & The most popular dishes are taken first & Take the order quantity by choosing the dish as the weight \\
No. 4 & The most urgent dishes are taken first & Take the reciprocal of the remaining time as the weight \\
\hline
\end{tabular}

The logic of the double-layer model is shown in Figure 3. After confirming the order information and preprocessing the dish-package, the restaurant can schedule the cooking plan with four different scheduling strategies. The upper model calls the package scheduling data from the bottom layer model. Finally, the optimal model is obtained after comparing the actual results using these strategies. Notably, the weight value method given in Table 1 is an approximate scheme for the optimal goal, wherein the final decision should be obtained only after four attempts.

Switch time may be applied for cooking different kinds of dish-packages. We found a similarity between the dish-package scheduling model and the VRP model when switch time is considered. Details about the similarity are analyzed in Section 5. However, the new VRP based model will not change the structure of the double-layer model. Only the bottom layer scheduling model for the dish-package is replaced. 
THE DOUBLE-LAYER MODEL

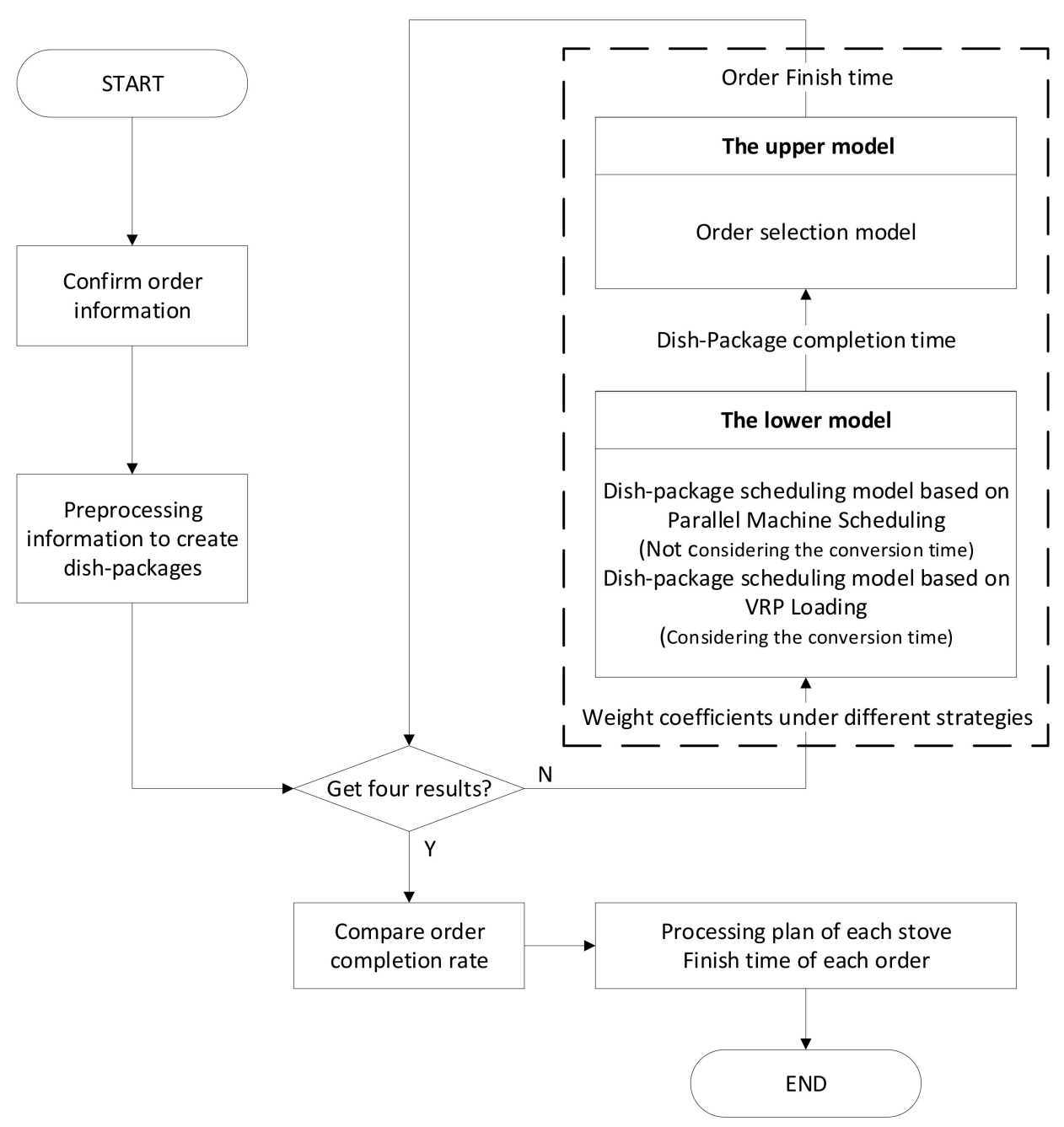

Figure 3. Logic of the double-layer model.

\subsection{The Lower Layer Model: Dish-Package Scheduling Model}

The problem is defined as follows.

1. Dish-packages $b$ are processed in the package set $B$. The cooking time and weight coefficient of dish-package $i$ is denoted as $C T_{i}$ and $a_{i}$.

2. Identical parallel processing stoves $m$ can be found in stove set $M$.

3. Switching time is ignored.

4. A time axis is set for each stove and non-overlapping time-slots are divided on this axis by applying the idea of virtual time-slot in parallel machine scheduling. The length of time slot is determined by the dish-package actually produced. The preset number of virtual time-slots as $b$ is set, which means that a dish-package must choose a virtual time slot from every stove. If the dish-package is not actually cooked on that stove, the completion time of this time slot is equal to its start time. The start time of the $i$ th time-slot on stove $k$ is denoted as $S T_{k i}$, and completion time is $F T_{k i}$.

The mathematical formulation of dish-package scheduling is given as:

$$
\operatorname{Min} F=\sum_{k=1}^{m} \sum_{i=1}^{b} F T_{k i} a_{i}
$$

Subject to:

$$
F T_{k i}=S T_{k i}+C T_{i} X_{k i}, \forall i \in B, \forall k \in M
$$




$$
\begin{gathered}
\left(S T_{k j}-F T_{k i}\right)\left(S T_{k i}-F T_{k j}\right) \leq 0, \forall i, j \in B, \forall k \in M \\
\sum_{k=1}^{m} X_{k i}=1, \forall i \in B, \forall k \in M \\
X_{k i}=\left\{\begin{array}{c}
\text { 1, package is assigned to the time slot ion stove } k \\
\text { 0, otherwise }
\end{array}, \forall i \in B, \forall k \in M\right.
\end{gathered}
$$

- Equation (1) is the objective function, which minimizes the weighted completion time of all dish-packages.

- Constraint (2) determines the completion time for each virtual timeslot. The completion time is equal to the start time in addition to processing time if there it is an actual processing task. Otherwise, the completion is equal to start time.

- The start time and completion time of $i$ th time-slot on stove $k\left[S T_{k i}, F T_{k i}\right]$ are given. Constraint (3) indicates that all time intervals cannot intersect. The joint result of this constraint and the objective function ensures that the completion time of the previous task is equal to the start time of the next task.

- Constraint (4) ensures that each dish-package must be actually processed.

- Constraint (5) denotes the binary restriction of the decision variable.

\subsection{The Upper Layer Model: Order Selection Model}

The order selection problem can be defined as follows:

1. Dish-packages $b$ can be found in the package set $B$. The actual completion time and weight coefficient of the dish-package $i$ is denoted by $T_{i}$ and $C_{i}$, respectively.

2. Orders $w$ can be found in the order set $W$. The actual completion time and the latest required time of the order $s$ is denoted as $T_{S}$ and $L_{s}$.

3. There are $p$ types of dishes in dish set $P$. The number of servings for dish type $p$ from order $s$ is $a_{s p}$. The subordinate relationship between dish-package $i$ and dish type $p$ is expressed by $d_{i p}$. If the dish type of the dish-package $i$ is dish type $p$, then $d_{i p}=1$, otherwise $d_{i p}=0$.

The mathematical formulation of the order selection problem is:

$$
\operatorname{Min} T=\sum_{i=1}^{b} T_{s} .
$$

Subject to:

$$
\begin{gathered}
\sum_{i=1}^{b} y_{s i} d_{i p} \geq a_{s p}, \forall i \in B, \forall s \in W, \forall p \in P \\
\sum_{s=1}^{w} y_{s i} \leq C_{i}, \forall i \in B, \forall s \in W \\
T_{s} \geq T_{i} x_{s i}, \forall i \in B, \forall s \in W \\
\sum_{s=1}^{w} Z_{s} \leq 30 \% w, \forall s \in W \\
Z_{s}=\left\{\begin{array}{c}
1, \text { order overtime, which is } T_{s}>L_{s} \\
0, \text { otherwise }
\end{array}, \forall s \in W\right. \\
x_{s i}=\left\{\begin{array}{c}
1, \text { order } s \text { choosign dish package } i \\
0, \text { otherwise } \\
10 x_{s i} \geq y_{s i}, \forall i \in B, \forall s \in W
\end{array}, \forall s \in W, \forall i \in B\right.
\end{gathered}
$$

- Equation (6) is the objective function that minimizes the completion time of each order.

- Constraint (7) indicates that all dishes from each order can be found from the corresponding dish-package. 
- Constraint (8) ensures that the total size of dishes selected in a dish-package cannot exceed its capacity.

- Constraint (9) ensures that the completion time of the order is determined by the completion time of its dish.

- Constraints (10) and (11) denote the binary restriction of $Z_{s}$, which is used to calculate the number of delayed orders. The restaurant should ensure service quality by controlling the delay proportion of orders, which is tentatively set as $30 \%$.

- Constraints (12) and (13) denote the binary restriction of $x_{s i}$, which indicates whether the dish package is selected for the order; $y_{s i}$ gives the number of servings of dishpackage $i$ selected by order $s$.

\section{Numerical Experiment and Results}

\subsection{Basic Data}

A restaurant needs to fulfill 10 online orders, and the menu of the restaurant includes 10 dishes. Each order has the expected meal conversion time. The information on the 10 orders is shown in Table 2.

Table 2. Order Information.

\begin{tabular}{cccccccccccc}
\hline Order No. & Expected Time/min & Dish 1 & Dish 2 & Dish 3 & Dish 4 & Dish 5 & Dish 6 & Dish 7 & Dish 8 & Dish 9 & Dish 10 \\
\hline $1^{\prime}$ & 20 & 1 & 0 & 0 & 0 & 0 & 0 & 0 & 0 & 1 \\
$2^{\prime}$ & 23 & 0 & 0 & 2 & 0 & 0 & 0 & 0 & 0 & 2 & 0 \\
$3^{\prime}$ & 27 & 0 & 3 & 0 & 4 & 0 & 0 & 0 & 1 & 0 & 0 \\
$4^{\prime}$ & 30 & 1 & 0 & 0 & 1 & 0 & 1 & 0 & 0 & 0 \\
$5^{\prime}$ & 31 & 2 & 0 & 0 & 0 & 0 & 0 & 0 & 0 & 0 \\
$6^{\prime}$ & 32 & 0 & 0 & 0 & 1 & 0 & 0 & 1 & 0 & 0 \\
$7^{\prime}$ & 38 & 1 & 0 & 1 & 0 & 0 & 0 & 0 & 0 & 0 \\
$8^{\prime}$ & 40 & 0 & 0 & 0 & 0 & 0 & 0 & 0 & 0 & 1 \\
$9^{\prime}$ & 42 & 1 & 0 & 0 & 0 & 0 & 0 & 0 & 0 & 1 \\
$10^{\prime}$ & 45 & 0 & 0 & 0 & 0 & 0 & 1 & 1 & 0 & 0 \\
\hline
\end{tabular}

The cooking time and the package limitation to each dish are shown in Table 3.

Table 3. Dish Information.

\begin{tabular}{ccccccccccc}
\hline Parameter & Dish 1 & Dish 2 & Dish 3 & Dish 4 & Dish 5 & Dish 6 & Dish 7 & Dish 8 & Dish 9 & Dish 10 \\
\hline Cooking time/min & 7 & 6 & 6.5 & 5.5 & 5 & 5 & 4.5 & 6.5 & 4.5 & 4 \\
Package limitation/portion & 2 & 2 & 1 & 3 & 2 & 3 & 2 & 2 & 3 & 1 \\
\hline
\end{tabular}

According to Figure 1, the order will be preprocessed first. Eighteen dish-packages are formed after merging. The dish-package information is obtained from Table 1, as shown in Table 4:

Table 4. Batch Information.

\begin{tabular}{|c|c|c|c|c|c|c|c|c|}
\hline $\begin{array}{c}\text { Package } \\
\text { No. }\end{array}$ & $\begin{array}{l}\text { Expected } \\
\text { Time/min }\end{array}$ & Dish Type & Servings/Piece & $\begin{array}{c}\text { Processing } \\
\text { Time/min }\end{array}$ & $\stackrel{a_{-}}{\text {Strategy I }}$ & $\stackrel{a_{-}}{\text {Strategy II }}$ & $\stackrel{a_{-}}{\text {Strategy III }}$ & $\stackrel{a_{-}}{\text {Strategy IV }}$ \\
\hline $1^{\prime \prime}$ & 20 & Dish 1 & 2 & 7 & 1 & 0.143 & 5 & 0.05 \\
\hline $2^{\prime \prime}$ & 31 & Dish 1 & 2 & 7 & 1 & 0.143 & 5 & 0.032 \\
\hline $3^{\prime \prime}$ & 38 & Dish 1 & 2 & 7 & 1 & 0.143 & 5 & 0.026 \\
\hline $4^{\prime \prime}$ & 27 & Dish 2 & 2 & 6 & 1 & 0.167 & 1 & 0.037 \\
\hline $5^{\prime \prime}$ & 27 & Dish 2 & 1 & 6 & 1 & 0.167 & 1 & 0.037 \\
\hline $6^{\prime \prime}$ & 23 & Dish 3 & 1 & 6.5 & 1 & 0.154 & 2 & 0.043 \\
\hline $7^{\prime \prime}$ & 23 & Dish 3 & 1 & 6.5 & 1 & 0.154 & 2 & 0.043 \\
\hline $8^{\prime \prime}$ & 38 & Dish 3 & 1 & 6.5 & 1 & 0.154 & 2 & 0.026 \\
\hline $9^{\prime \prime}$ & 27 & Dish 4 & 3 & 5.5 & 1 & 0.182 & 3 & 0.037 \\
\hline $10^{\prime \prime}$ & 27 & Dish 4 & 3 & 5.5 & 1 & 0.182 & 3 & 0.037 \\
\hline $11^{\prime \prime}$ & 30 & Dish 6 & 2 & 5 & 1 & 0.2 & 2 & 0.033 \\
\hline $12^{\prime \prime}$ & 32 & Dish 7 & 2 & 4.5 & 1 & 0.222 & 2 & 0.031 \\
\hline $13^{\prime \prime}$ & 27 & Dish 8 & 1 & 6.5 & 1 & 0.154 & 1 & 0.037 \\
\hline $14^{\prime \prime}$ & 20 & Dish 9 & 3 & 4.5 & 1 & 0.222 & 4 & 0.05 \\
\hline
\end{tabular}


Table 4. Cont.

\begin{tabular}{|c|c|c|c|c|c|c|c|c|}
\hline $\begin{array}{c}\text { Package } \\
\text { No. }\end{array}$ & $\begin{array}{l}\text { Expected } \\
\text { Time/min }\end{array}$ & Dish Type & Servings/Piece & $\begin{array}{l}\text { Processing } \\
\text { Time/min }\end{array}$ & $\stackrel{a_{-}}{\text {Strategy I }}$ & $\stackrel{a_{-}}{\text {Strategy II }}$ & $\stackrel{a_{-}}{\text {Strategy III }}$ & $\frac{a_{-}}{\text {Strategy IV }}$ \\
\hline $15^{\prime \prime}$ & 40 & Dish 9 & 2 & 4.5 & 1 & 0.222 & 4 & 0.025 \\
\hline $16^{\prime \prime}$ & 32 & Dish 10 & 1 & 4 & 1 & 0.25 & 2 & 0.031 \\
\hline $17^{\prime \prime}$ & 40 & Dish 10 & 1 & 4 & 1 & 0.25 & 2 & 0.025 \\
\hline $18^{\prime \prime}$ & 40 & Dish 10 & 1 & 4 & 1 & 0.25 & 2 & 0.025 \\
\hline
\end{tabular}

\subsection{Result}

The numerical experiment is solved in Lingo 17.0, and the feasible solution can be obtained in reasonable calculation time. The results are shown in Table 5 after comparing the four strategies.

Table 5. Results of scheduling model.

\begin{tabular}{|c|c|c|c|c|}
\hline Evaluating Indicator & Strategy I & Strategy II ${ }^{1}$ & Strategy III & Strategy IV \\
\hline The number of delayed orders & 3 & 4 & 3 & 3 \\
\hline The total delayed time of the orders $/ \mathrm{min}$ & 19.5 & 25.5 & 25 & 17.5 \\
\hline
\end{tabular}

${ }^{1}$ Solution can be obtained after relaxing the constraint.

In this experiment, Strategy IV has the least total delayed time. Strategy II cannot obtain a feasible solution until the constraint that more than $60 \%$ of the order should be finished on time is relaxed. To explore the details, the scheduling result of the bottom layer dish-package is analyzed, as shown in Table 6.

Table 6. Result analysis of the scheduling model based on parallel machine scheduling.

\begin{tabular}{|c|c|c|c|c|}
\hline Evaluating Indicator & Strategy I & Strategy II & Strategy III & Strategy IV \\
\hline The number of delayed dish-packages & 3 & 4 & 4 & 2 \\
\hline The total delayed time of dish-packages/min & 19.5 & 30 & 22 & 11 \\
\hline Sum of dish-packages finish time/min & 321 & 323.5 & 353.5 & 336 \\
\hline Max stoves' completion time gap $/ \mathrm{min}$ & 1.5 & 5.5 & 10 & 7 \\
\hline Total stove completion time's difference/min & 2 & 6 & 14 & 8 \\
\hline
\end{tabular}

Optimal Strategy IV minimizes time delay in the bottom layer model. The cooking arrangement of 18 dish packages on the stove is shown in Figure 4. Although the number of delayed dish-packages are four in both Strategy II and Strategy III, the total time delay is longer in Strategy II, which directly breaks the constraint. In addition, the "Stoves" completion time difference" of Strategy IV is larger than that of Strategy I. This result indicates that the working time of different stoves in Strategy IV is not well balanced. Therefore, employees should be allocated between stoves at the same time to ensure that the chef's workload is balanced.

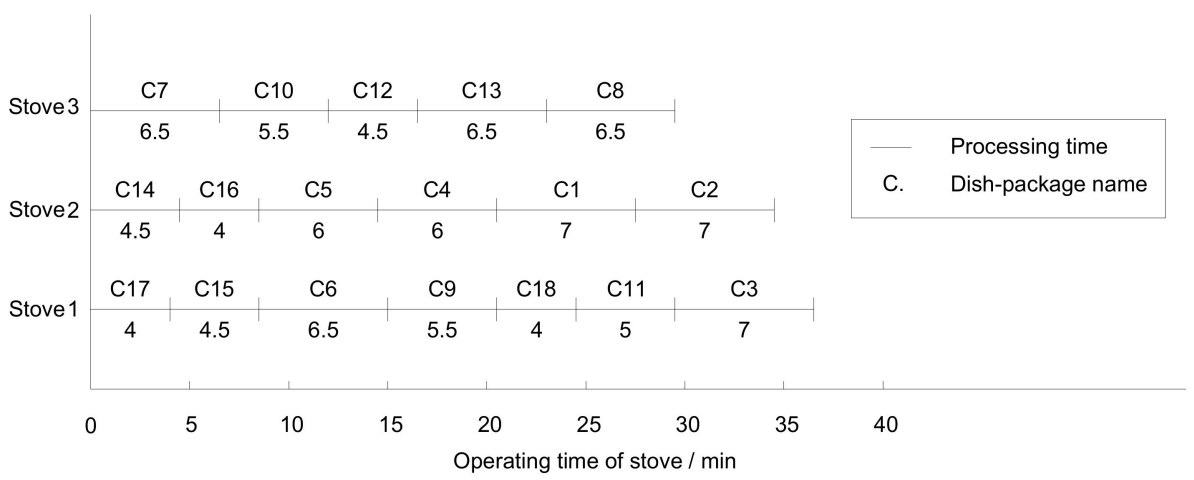

Figure 4. Gantt chart of processing under Strategy IV. 


\section{Model Expansion}

\subsection{Dish-Package Scheduling Model While Considering Switching Time}

This problem is essentially similar to VRP if the switching time between the dishpackages is considered. First, each dish-package is assumed to be a customer, and the processing time of the dish-package is the loading and unloading time of the goods at the customer's location. Second, the stoves can be likened to the vehicles in a VRP model. The meal scheduling of the dish-packages focuses on where the dishes are cooked and in what sequence. This situation is similar in VRP, which also focuses on how customer demand is satisfied with optimal vehicles and sequences. Third, the switching time of the packages is similar to the transportation time in the VRP problem.

The extended cooking problem can be defined as follows:

1. A virtual dish-package $i=0$ is imported, and then turned into a problem with $b+1$ dish-packages, which are processed in the new package set $B \prime$. The processing time and weight coefficient of dish-package $i=0$ is denoted by $C T_{i}=0$ and $a_{i}=0$. The virtual dish-package is introduced to replicate the stove scheduling model after the basic VRP model. Thus, the existing VRP algorithms can help to solve the stove scheduling model.

2. There are $m$ identical parallel processing stoves in stove set $M$.

3. The start time and the finish time of the $i$ th dish-package on stove $k$ is denoted by $S T_{k i}$ and $F T_{k i}$, respectively.

The mathematical formulation of the extended cooking problem is:

$$
\operatorname{Min} T=\sum_{k=1}^{m} \sum_{i=0}^{n} F T_{k i} a_{i} .
$$

Subject to:

$$
\begin{gathered}
\sum_{i=1}^{n} X_{0 i k}=1, \forall i \in B^{\prime}, i \neq 0, \forall k \in M \\
\sum_{i=1}^{n} X_{i 0 k}=1, \forall i \in B^{\prime}, i \neq 0, \forall k \in M \\
\sum_{j=1}^{n} X_{j i k}=Y_{k i}, \forall\{i, j\} \in B^{\prime}, j \neq i, i \neq 0, \forall k \in M \\
\sum_{j=1}^{n} X_{j i k} \geq 1, \forall\{i, j\} \in B^{\prime}, j \neq i, i \neq 0, \forall k \in M \\
\sum_{j=1}^{n} X_{i j k} \geq 1, \forall\{i, j\} \in B^{\prime}, j \neq i, i \neq 0, \forall k \in M \\
\sum_{j=1}^{n} X_{i j k}=\sum_{j=1}^{n} X_{j i k}, \forall\{i, j\} \in B^{\prime}, j \neq i, i \neq 0, \forall k \in M \\
\sum_{k=1}^{m} Y_{k i}=1, \forall i \in B^{\prime}, i \neq 0, \forall k \in M \\
F T_{k i}=S T_{k i}+C T_{i}, \forall i \in B^{\prime}, i \neq 0, \forall k \in M \\
0, \text { when } X_{0 i k}=1 \\
X_{i j k}=\left\{\begin{array}{c}
\sum_{k i}=\text { cook package } j \text { after } i \text { on stove } k \\
0, \text { otherwise }
\end{array}, \forall\{i, j\} \in B^{\prime}, i \neq 0, \forall k \in M\right.
\end{gathered}
$$




$$
Y_{k i}=\left\{\begin{array}{c}
1, \text { dish package } i \text { processed on stove } k \\
0, \text { otherwise }
\end{array}, \forall i \in B^{\prime}, \forall k \in M\right.
$$

- Equation (14) is the objective function, which minimizes the weighted finish time of the dish-packages.

- Constraints (15) and (16) ensure that every stove should start and finish on the virtual dish-package $i=0$.

- Constraint (17) determines the relationship between the decision variable $X_{j i k}$ and $Y_{k i}$.

- Constraints (18)-(20) ensure that each dish-package can only choose one stove for processing.

- Constraint (21) ensures each dish-package must be processed.

- Constraint (22) determines that the finish time of dish-package $i$ on stove $k$ is equal to its start time and processing time.

- Constraint (23) determines the start time of the dish-package is equal to the completion time and switching time in the previous package.

- Constraints (24) and (25) denote the binary restriction of the decision variable $X_{j i k}$ and $Y_{k i}$.

\subsection{Supplementary Data}

Compared with the original data, the switching time between dish-packages needs to be switched for the numerical experiment. The switch time is related to factors, such as food characteristics, stove cleaning time, food ingredients' difference, and other related factors.

In our experiment, we assume that the switch time after dish 1 is $2 \mathrm{~min}$. The switch time after dishes 6 and 7 is $3 \mathrm{~min}$. The remaining dish switch time is $1 \mathrm{~min}$. Switch time is not required for packages of the same dish type. The switch time is 0 for virtual dishpackage 0 . A switch time matrix of $19 \times 19$ is obtained due to length limitations. The detail of the matrix is not presented.

The original expected finish time is delayed by $6 \mathrm{~min}$.

\subsection{Result}

We also use Lingo 17.0 to solve the experiment. A feasible solution is obtained at an acceptable calculation time. The results are shown in Table 7, after solving the double-layer scheduling model.

Table 7. Results when switch time is considered.

\begin{tabular}{ccccc}
\hline Evaluating Indicator & Strategy I & Strategy II & Strategy III & Strategy IV \\
\hline Number of delayed orders & 1 & 3 & 1 & 3 \\
Total time delay of orders/min & 9 & 16.5 & 10.5 & 18.5 \\
\hline
\end{tabular}

In the extended experiment, Strategy I performed the best. The details of the results in the lower-layer model are shown in Table 8.

Table 8. Result analysis of scheduling model based on VRP.

\begin{tabular}{ccccc}
\hline Evaluating Indicator & Strategy I & Strategy II & Strategy III & Strategy IV \\
\hline Number of delayed dish-packages & 2 & 4 & 4 & 3 \\
Total time delay of dish-packages $/$ min & 11 & 20.5 & 18 & 15 \\
Total dish-package finish time/min & 386.5 & 377 & 411 & 396 \\
Max stoves' completion time gap/min & 8 & 2.5 & 8 & 8.5 \\
Total stove completion time's difference/min & 8.33 & 2.67 & 8.67 & 9.33 \\
\hline
\end{tabular}

The production sequence arrangement of 18 dish packages on the stove is shown in 


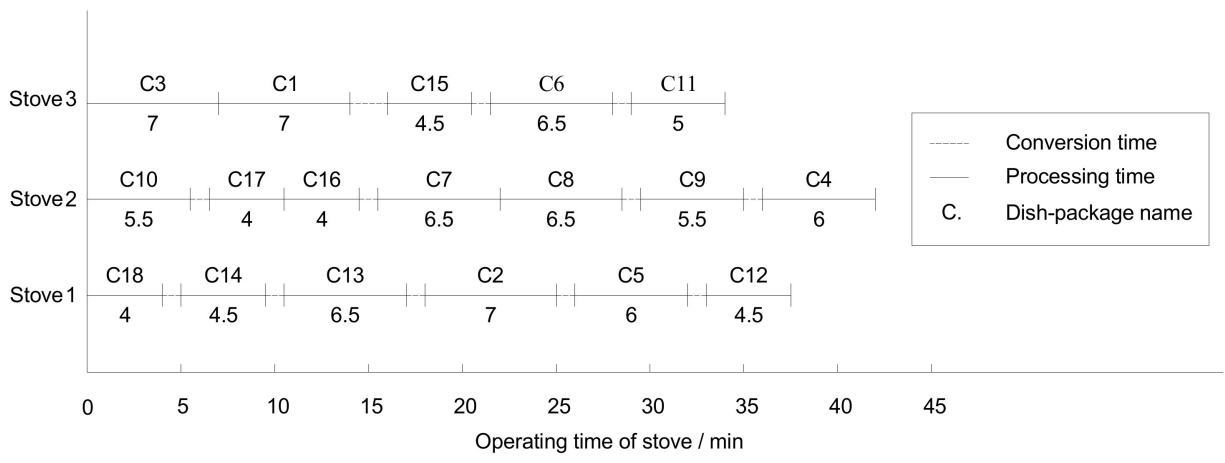

Figure 5. Gantt chart of processing under Strategy 1.

\section{Conclusions}

The present paper aims to improve meal cooking efficiency in the on-demand food delivery industry. A double-layer multi-stove meal scheduling model with mergeable products is constructed. Four strategies for calculating the weight coefficients of the dishpackage are designed. The switch time is ignored in the bottom layer of the basic model. A dish-package processing model based on parallel machine scheduling is established. Furthermore, a model based on VRP is established when the switch time is considered. Different conclusions are obtained among the four scheduling strategies based on comparative analysis involving the bottom and upper layer results. The results show that "the most emergent first" strategy is the best when switch time is ignored. By contrast, the "workload balance" strategy performs best when the switching time is considered. These results provide strong evidence on the necessity of using the double-layer model for multi-strategy comparison in an actual meal scheduling problem.

\section{Future Research}

Future research should examine the algorithms for a large-scale problem and a mixed model considering both for online and offline orders.

Author Contributions: Conceptualization, T.D. and X.F.; methodology, T.D. and X.F.; software, X.F.; validation, X.F writing—original draft preparation, X.F.; writing—review and editing, T.D. All authors have read and agreed to the published version of the manuscript.

Funding: This work was supported by the National Natural Science Foundation of China (Project No.: 71872037).

Conflicts of Interest: The authors declare no conflict of interest.

\section{References}

1. Zhang, C.; Jiang, J.; Jin, H.; Chen, T. The Impact of COVID-19 on Consumers' Psychological Behavior Based on Data Mining for Online User Comments in the Catering Industry in China. Int. J. Environ. Res. Public Health 2021, 18, 4178. [CrossRef] [PubMed]

2. Liu, Y.; Guo, B.; Du, H.; Yu, Z.; Zhang, D.; Chen, C. Poster: FooDNet. In Proceedings of the 23rd Annual International Conference on Mobile Computing and Networking, MobiCom '17, Snowbird, UT, USA, 16-20 October 2017; pp. 564-566. [CrossRef]

3. Liu, P.; Lv, J.; Jiang, T.; Chai, X. Equilibrium Joining Strategies of Delay-Sensitive Customers in a Queueing System with Service Quality Feedback. Discret. Dyn. Nat. Soc. 2020, 2020, 5906407. [CrossRef]

4. Naderi, B.; Roshanaei, V. Branch-Relax-and-Check: A tractable decomposition method for order acceptance and identical parallel machine scheduling. Eur. J. Oper. Res. 2020, 286, 811-827. [CrossRef]

5. Brauner, N.; Kovalyov, M.Y.; Quilliot, A.; Toussaint, H. No-idle parallel-machine scheduling of unit-time jobs with a small number of distinct release dates and deadlines. Comput. Oper. Res. 2021, 132, 105315. [CrossRef]

6. Tighazoui, A.; Sauvey, C.; Sauer, N. Minimizing the Total Weighted Waiting Times and Instability in a Rescheduling Problem with Dynamic Jobs Weight. Appl. Sci. 2021, 11, 7040. [CrossRef]

7. Kim, J.; Kim, H.-J. Parallel machine scheduling with multiple processing alternatives and sequence-dependent setup times. Int. J. Prod. Res. 2020, 59, 5438-5453. [CrossRef]

8. Yepes-Borrero, J.C.; Perea, F.; Ruiz, R.; Villa, F. Bi-objective parallel machine scheduling with additional resources during setups. Eur. J. Oper. Res. 2021, 292, 443-455. [CrossRef] 
9. Lee, J.-H.; Jang, H. Uniform Parallel Machine Scheduling with Dedicated Machines, Job Splitting and Setup Resources. Sustainability 2019, 11, 7137. [CrossRef]

10. Hidri, L.; Alqahtani, A.; Gazdar, A.; Ben Youssef, B. Green Scheduling of Identical Parallel Machines with Release Date, Delivery Time and No-Idle Machine Constraints. Sustainability 2021, 13, 9277. [CrossRef]

11. Dong, J.; Goebel, R.; Hu, J.; Lin, G.; Su, B. Minimizing total job completion time in MapReduce scheduling. Comput. Ind. Eng. 2021, 158, 107387. [CrossRef]

12. Witteman, M.; Deng, Q.; Santos, B.F. A bin packing approach to solve the aircraft maintenance task allocation problem. Eur. J. Oper. Res. 2021, 294, 365-376. [CrossRef]

13. De Lima, V.L.; Alves, C.; Clautiaux, F.; Iori, M.; Valério de Carvalho, J.M. Arc flow formulations based on dynamic programming: Theoretical foundations and applications. Eur. J. Oper. Res. 2022, 296, 3-21. [CrossRef]

14. Garrisi, G.; Cervelló-Pastor, C. Train-Scheduling Optimization Model for Railway Networks with Multiplatform Stations. Sustainability. 2020, 12, 257. [CrossRef]

15. Psychas, K.; Ghaderi, J. High-Throughput Bin Packing: Scheduling Jobs With Random Resource Demands in Clusters. IEEE/ACM Trans. Netw. 2021, 29, 220-233. [CrossRef]

16. Dantzig, G.B.; Ramser, J.H. The Truck Dispatching Problem. Manag. Sci. 1959, 6, 80-91. [CrossRef]

17. Zhang, H.; Ge, H.; Yang, J.; Tong, Y. Review of Vehicle Routing Problems: Models, Classification and Solving Algorithms. Arch. Comput. Methods Eng. 2021, 1-27. [CrossRef]

18. Baldacci, R.; Mingozzi, A.; Roberti, R. Recent exact algorithms for solving the vehicle routing problem under capacity and time window constraints. Eur. J. Oper. Res. 2012, 218, 1-6. [CrossRef]

19. Chen, C.; Demir, E.; Huang, Y. An adaptive large neighborhood search heuristic for the vehicle routing problem with time windows and delivery robots. Eur. J. Oper. Res. 2021, 294, 1164-1180. [CrossRef]

20. Fallahtafti, A.; Karimi, H.; Ardjmand, E.; Ghalehkhondabi, I. Time slot management in selective pickup and delivery problem with mixed time windows. Comput. Ind. Eng. 2021, 159, 107512. [CrossRef] 